\title{
Common origin of the satellite DNAs of the Hawaiian spiders of the genus Tetragnatha: evolutionary constraints on the length and nucleotide composition of the repeats
}

\author{
Joan Pons*, Rosemary G. Gillespie \\ Division of Insect Biology, ESPM, University of California, Wellman Hall 201 Berkeley, CA 94720-3112, USA
}

Received 19 February 2003; received in revised form 6 April 2003; accepted 7 April 2003

Received by D. Fitch

\begin{abstract}
The present study characterizes the satellite DNA sequences of three endemic Hawaiian spiders, Tetragnatha acuta, Tetragnatha hawaiensis, and Tetragnatha quasimodo, to test the degree of conservation of these sequences within a closely related group of arthropods. The length and nucleotide composition of repeats of the three species is very similar both at intra- and inter-specific level. However, their nucleotide sequence is very divergent at the inter-specific level although conserve substantial similarity in some stretches. These results suggest a common origin of the Tetragnatha satellite DNAs and evolutionary constraint in the length and the nucleotide composition of these repeats at the inter-specific level but not in their nucleotide sequences. At the intra-specific level, the three species show a different degree of sequence identity between repeats as a result of specific historical processes. Tetragnatha hawaiensis shows a strong homogenization of the monomeric sequences. Tetragnatha quasimodo also shows strong homogenization but the actual repeats are higher-order repeats (dimers linking two divergent subfamilies of monomers). On the other hand, the existence of the three subfamilies of repeats in $T$. acuta, showing divergent sequence identity, both within and between subfamilies, suggests low homogenization of the repeats. Finally, evidence of gene conversion or unequal sister chromatid exchange events in $T$. quasimodo and $T$. acuta suggests that recombination is involved in the spreading and homogenization of stDNA sequences.
\end{abstract}

(C) 2003 Elsevier Science B.V. All rights reserved.

Keywords: Repetitive DNA; Gene conversion; Higher-order repeat

\section{Introduction}

Satellite DNA (stDNA) is a highly repetitive DNA organized in long tandem repeats in constitutive heterochromatin (Charlesworth et al., 1994). Its function is still unclear but many studies associate satellite DNA with heterochromatin organization and centromeric function (Ugarkovic and Plohl, 2002). Different motifs found in satellite repeats are thought to play an important role in those functions: phased $\mathrm{A}$ or $\mathrm{T}$ runs $\geq 3$ producing DNA

Abbreviations: bp, base pair(s); F81 + I, Felsenstein 1981 model plus invariant sites; HKY, Hasegawa-Kishino-Yano 1985 model; hLRT, hierarchical likelihood ratio test; HOR(s), higher-order repeat(s); $\operatorname{tDNA}(\mathrm{s})$, satellite DNA(s).

* Corresponding author. Present address: The Natural History Museum, Department of Entomology, Cromwell Road, London SW7 5B, UK. Tel.: +44-207-942-5048; fax: + 44-207-942-5229.

E-mail address: joap@nhm.ac.uk (J. Pons). bending (Martinez-Balbas et al., 1990), cruciform configurations (Juan et al., 1993) or the presence of specific motifs binding centromeric proteins (Lorite et al., 2002). Concerted evolution generally leads to striking intra-specific sequence identity among repetitive units, which constitute a stDNA family (Elder and Turner, 1995). However, some stDNA families have divergent repetitive sequences that can be grouped into different subfamilies due to the presence of diagnostic sites (Trick and Dover, 1984; Pons et al., 2002a). These positions have a particular mutation across all sequences of one subfamily, while at the same sites all sequences of the other subfamily have a different mutation (homogenized sites). In some species, monomers from different subfamilies are linked to form a higher-order repeat (HOR, Willard and Wayne, 1987; Pons et al., 2002b). Thus, the different monomers within a HOR show substantial sequence divergence, because they are from divergent subfamilies, but have striking sequence identity 
between HORs. For instance, in human chromosome 7 there are two HORs based on different subfamilies of the $\sim 171$ bp alphoid monomer: a 6-monomer HOR, and a dimer (Willard and Wayne, 1987).

On the other hand, at the inter-specific level concerted evolution generally leads to species-specific stDNAs whose repeats are completely different in length and nucleotide sequence. The concerted evolution of repetitive sequences is driven by the gradual spreading of new repeat units by unequal transfer, and particularly by the redistribution of chromosomes through sexual reproduction (Dover, 1986; Mantovani et al., 1997). This evolutionary dynamic leads to homogenization of the repetitive units through a particular stDNA family and fixation through the whole population. Factors involved in concerted evolution include the frequency of unequal transfer between chromosomes (unequal crossing over, gene conversion, and transposition by rolling circle amplification), the number of repetitive units, and population size. Any bias in these factors or a higher mutation rate would lead to a lower degree of intraspecific sequence identity among repeats (Dover, 1986; Stephan and Cho, 1994).

Although there are a few examples of a group of species sharing similar stDNA sequences (e.g. Modi et al., 1996; Pons et al., 2002a; Lorite et al., 2002), study of the evolutionary dynamics of stDNA sequences at the interspecific level is usually not possible because of the absence of similarity. Exceptions to this are studies of stDNA sequences from very closely related species originating from adaptive radiation on islands, in particular Hawaiian Drosophila (Miklos and Gill, 1981) and Canarian darkling Pimelia beetles (Pons et al., 2002a). Previous work on the development of AFLP markers in the endemic Hawaiian Tetragnatha quasimodo spider unveiled the characteristic conspicuous ladder of oligomers of stDNAs when its genomic DNA was digested with the restriction enzyme Bfa I and subsequently electrophoresed on an agarose gel (J. Pons and R.G. Gillespie, unpublished). Here we test the conservation of stDNA sequences within closely related arthropods, endemic Hawaiian Tetragnatha spiders (Araneae: Tetragnathidae). We compare stDNA sequences, both at intra- and inter-specific levels, from one species of each of the three major Hawaiian Tetragnatha clades which have at least two independent origins (Gillespie et al., 1994). We characterize in detail Tetragnatha hawaiensis Simon, 1900, T. quasimodo Gillespie, 1991 from the cursorial 'spiny leg' clade, and T. acuta Gillespie, 1994 from a clade of webbuilding species.

\section{Material and methods}

\subsection{Sampling and genomic DNA isolation}

Individuals were collected in different Hawaiian localities: T. quasimodo from Mount Kaala (600 m),
Oahu, T. acuta from Kaupo (2000 m), Maui, and T. hawaiensis from Kahili (650 m), Kauai. For each species, DNA was isolated from frozen adult specimens stored below $-80{ }^{\circ} \mathrm{C}$ by standard phenol extraction and ethanol precipitation procedures.

\subsection{Isolation, cloning and sequencing of satellite DNA}

One to two micrograms of genomic DNA were digested with restriction enzymes according to the instructions of the manufacturer (Roche, New England), and the fragments obtained were electrophoresed on $2 \%$ agarose gels containing ethidium bromide. Several restriction enzymes were tested for isolation of stDNA sequences: Acc I, Afl II, Alu I, Apa I, Bfa I, Bst BI, Dra I, Hae III, Hinc II, Hinf I, Mbo I, and Sal I. Conspicuous DNA bands of about 190 and 380 bp were found. These were cut from gel, purified with the Qiaquick Gel Extraction Kit (Qiagen), ligated into the Eco $R \mathrm{~V}$ site of the pMOSBlue vector (pMOSBlue blunt ended cloning kit, Amersham Pharmacia Biotech), and used to transform Sure ${ }^{\mathrm{TM}}$ competent cells (Stratagene). Clones were screened using the $\beta$-galactosidase gene blue-white color system. Recombinant clones were sequenced from both strands by the dideoxy sequencing method using the Big Dye $^{\mathrm{TM}}$ Terminator Cycle Sequencing Kit and the automatic sequencing system ABI PRISM ${ }^{\mathrm{TM}} 377$ DNA Sequencer (Applied Biosystems).

\subsection{Southern hybridizations}

After electrophoresis DNA genomic restriction fragments on agarose gels were denatured, transferred onto nylon membranes (Osmotics), and finally cross-linked to the membrane by exposure to ultraviolet light according to the standard Southern protocol. Cloned monomers, excised from the plasmid pMOSBlue using the restriction enzymes Nde I and Bam H I, were used as probes: pHAW02 (T. hawaiensis), pQUA05 (T. quasimodo), and pACU04 (T. acuta). Digoxigenin labeling of the probe, filter hybridizations, and detection of the hybridization signals were performed as described in the manual for the DIG High Prime DNA Labeling and Detection Starter Kit I (Roche). A similarity of $80-75 \%$ was allowed in the Southern hybridization (post-hybridization washes $2 \times 5 \mathrm{~min}$ in $2 \times$ SSC and $0.1 \%$ SDS at room temperature, and $2 \times 15 \mathrm{~min}$ in $0.1 \times \mathrm{SSC}$ and $0.1 \% \operatorname{SDS}$ at $37^{\circ} \mathrm{C}$ ).

\subsection{Estimation of stDNA percentages}

The percentage of stDNA in $T$. quasimodo and in T. hawaiensis was estimated by dot-blot hybridization. DNA was blotted onto membranes, denatured with $0.5 \mathrm{~N}$ $\mathrm{NaOH}$ and cross-linked to the membrane by exposure to ultraviolet light. The monomer pQUA05 was used as probe and stDNA dilutions in the T. quasimodo dot-blot, and the monomer pHAW02 in the T. hawaiensis dot-blot. The 
hybridization signals from six different amounts of stDNA (from 1 to $16 \mathrm{ng}$ ) were compared with the signals from six different amounts of genomic DNA (from 10 to $80 \mathrm{ng}$ ) to determine the percentage of the stDNA in the genome. Posthybridization washes were performed as outlined above for Southern hybridization (80-75\% similarity). The dot-blots were scanned and then analyzed with Quantitative One software v. 4.2.1 (BIO-RAD). The software estimates the percentage of stDNA in the genomic DNA by measuring the hybridization signal of the stDNA probe from the different genomic DNA spots using the stDNA standard curve (hybridization signals of the different amounts of stDNA). The relative amount of stDNA in T. acuta was determined from genomic DNA digested with HinfI and subsequently electrophoresed on an agarose gel. The digitalization and densitometric measurements of the gel were performed with Quantitative One software v. 4.2.1 (BIO-RAD). The percentage of stDNA was estimated by comparing the signal of conspicuous bands representing stDNA relative to the total DNA signal (Pons et al., 2002a).

\subsection{Sequence analysis}

For most of the analyses, stDNA sequences were analyzed as single repeats or monomers, and therefore the two repeats of each $T$. quasimodo and $T$. hawaiensis dimer were analyzed independently. The repeat having the restriction site used to isolate the dimer was named repeat A, and the repeat lacking that restriction site was called repeat B. Multiple sequence alignment was performed using the default parameters of Clustal W v. 1.7 (Higgins et al., 1996). Distance trees were built by the Neighbor-Joining method, and sequence divergences were calculated according to the maximum likelihood model of nucleotide evolution selected by hLRT in Modeltest v. 3.06 (Posada and Crandall, 1998). Parsimony trees were obtained after 1000 random addition searches with TBR branch swapping and under the 'Multrees' option. All nucleotide positions in the matrix, including gap positions, were considered equivalent and weighted equally. Bootstrap values were calculated on 1000 replicates in PAUP* 4.05 (Swofford, 2002). The nucleotide composition and homogeneity of base frequencies across sequences in the repetitive units were also estimated using $\chi^{2}$ tests in PAUP*. DNA polymorphism, DNA divergence, and putative gene conversion events were estimated using DnaSP v. 3.51 (Rozas and Rozas, 1997). Sequence divergence between subfamilies was calculated as the average nucleotide substitution per site $\left(D_{x y}\right.$ value from DnaSP 3.51 ), with 'subfamily' indicating a monophyletic group of repeats supported by high bootstrap values whose sequences exhibit substantially higher nucleotide identity within than between subfamilies and fixed mutations that are diagnostic of each subfamily. The distribution of nucleotide variability throughout the monomer sequence was analyzed using the sliding window command implemented in DNA polymorphism and DNA divergence between populations options of DnaSP 3.51 software (window length $=1$, step size $=1)$. The random sequences were generated with the program Shuffleseq (Rice et al., 2000) available on line from The Institute Pasteur's web page (http://www.bioweb. pasteur.fr/seqanal/interfaces/shuffleseq.html).

\section{Results}

\subsection{Detection, cloning and Southern blot of stDNAs}

Digestion of genomic DNA with the endonucleases $B f a$ I for T. quasimodo, Acc I for T. hawaiensis, and Hinf I for T. acuta, and subsequent analysis with agarose gel electrophoresis revealed conspicuous bands of about $190 \mathrm{bp}$ (all species) and $380 \mathrm{bp} \mathrm{(T.} \mathrm{quasimodo} \mathrm{and} \mathrm{T.} \mathrm{hawaiensis,}$ Fig. 1a). These conspicuous bands were cloned. Other restriction enzymes showed similar but weaker patterns of bands in the three species. Southern hybridization of the digested DNA of $T$. quasimodo with a cloned sequence of about $190 \mathrm{bp}$ as probe (pQUA05) showed a ladder of oligomers (Fig. 1b). Similar Southern hybridization in T. hawaiensis (probe pHAW02) and T. acuta (probe pACU02) revealed strong signal in the monomeric and dimeric bands only (Fig. 1c,d, respectively). These patterns are indicative of a tandem arrangement of highly repetitive DNA sequences in the genome of the three species with a repetitive unit of about $190 \mathrm{bp}$. The estimated percentage of the stDNA in the T. quasimodo genome was $25.61 \pm 2.94$ and in T. hawaiensis $30.38 \pm 7.06$ (Fig. 2). The percentage of the stDNA in $T$. acuta could not be estimated by dot blot but could roughly be estimated by densitometric analysis of digested genomic DNA $(19.12 \pm 1.45)$.

\subsection{Analysis of stDNA sequences}

\subsubsection{Intraspecific variability}

The NJ tree, inferred from best-fit model (HKY), shows
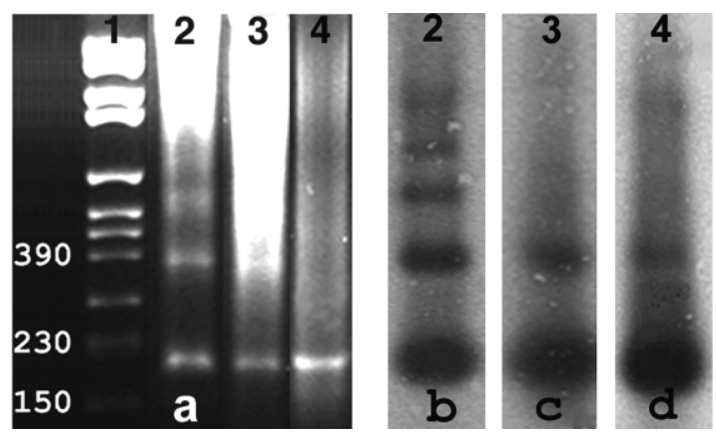

Fig. 1. (a) Agarose gel electrophoresis of the genomic DNA of T. quasimodo digested with Bfa I (lane 2), T. hawaiensis with Acc I (lane 3), and T. acuta with Hinf I (lane 4). Lane 1 shows the DNA molecular standard Marker VI from Roche whose bands range from $2.1 \mathrm{~Kb}$ to $150 \mathrm{bp}$. (b) Southern blot of the gel after hybridization using the monomer pQUA05 as a probe, (c) monomer pHAW02, and (d) monomer pACU04. 


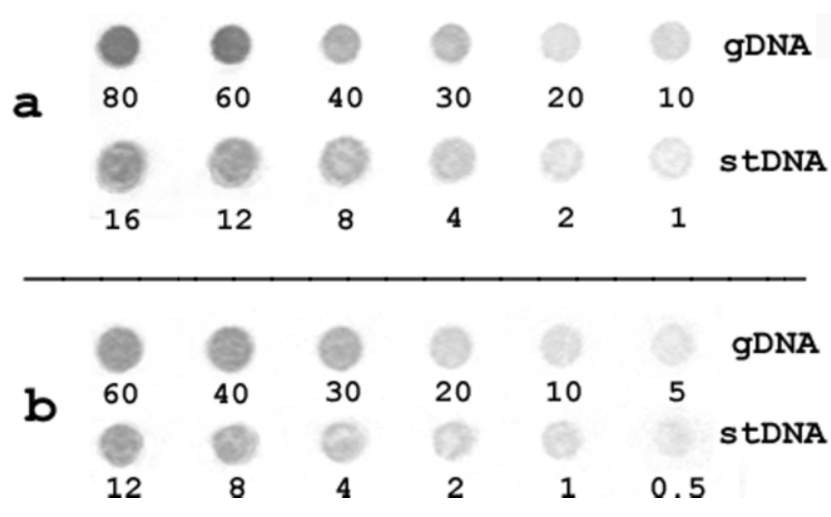

Fig. 2. Dot-blot hybridization results. (a) The monomer pQUA05 was used as stDNA control and probe for the quantification of the fraction of stDNA relative to genomic DNA in T. quasimodo. (b) The monomer pHAW02 was used for the quantification of that fraction in the genomic DNA of T. hawaiensis. Numbers indicate the amount of DNA spotted (in nanograms).

no indication of subfamilies in T. hawaiensis (Fig. 3). Repetitive units are $183 \mathrm{bp}$ long out of the sequence pHAW13 which has a length of $184 \mathrm{bp}$. The sequences of T. hawaiensis are homogeneous in their nucleotide composition (57.7\% $\mathrm{A}+\mathrm{T}$ rich on average). Tetragnatha hawaiensis sequences show low nucleotide diversity (0.028) that appears to be evenly distributed throughout the repetitive unit sequence. Half of this diversity is due to single nucleotide substitutions (autapomorphies, 37 out of 65 mutations), and the other fraction is mostly due to particular nucleotide substitutions shared within a particular nucleotide site and across several sequences (synapomorphies). For instance, seven sequences share a $G$ in the position 177 and four sequences share a $\mathrm{T}$ and an $\mathrm{A}$ in the positions 166 and 182, respectively.

The NJ tree inferred from the best-fit model (F81) reveals the presence of two groups of repetitive units supported by high bootstrap values in T. quasimodo (Fig. 4): subfamily QUA 1 (11 sequences) and subfamily QUA 2 (16 sequences). Similar results were obtained using parsimony. The length of the repeat units is very conserved, with the consensus sequence of both subfamilies $189 \mathrm{bp}$ long, though few units exhibit single base pair indels (pQUA20, pQUA01, pdQUA34A, pdQUA43A, and pQUA06). All $T$. quasimodo sequences proved to be homogeneous in their nucleotide composition (55.9\% $\mathrm{A}+\mathrm{T}$ rich in average). The subfamilies QUA 1 and QUA 2 show low nucleotide diversity, 0.026 and 0.030 , respectively, that appears to be evenly distributed throughout the monomer sequence. Most of these substitutions are autapomorphies (20 out of 22 mutations within the subfamily QUA 1 , and 30 out of 39 within the subfamily QUA 2) and the remainder synapomorphies.

The nucleotide divergence between subfamilies (0.096), though higher than within subfamilies, appears also to be evenly distributed throughout the repeat. Most of this divergence is due to diagnostic nucleotide substitutions: homogenized sites (positions 95, 96, 97, 98, 117, and 127), or mostly homogenized $(12,15,64,71,76,106,135,143$, 144,175 , and 177). Subfamilies also differ in two diagnostic indels (positions 124 and 142). The presence of diagnostic substitutions between subfamilies allows us to test for the presence of tracts in specific sequences resulting from gene conversion or unequal sister chromatid exchange. Four sequences show tracts with the diagnostic substitutions of the other subfamily possibly caused by gene conversion: sequences pQUA01 (positions 144-177), pQUA05 (64-76), pQUA25 (175-177) and pQUA30 (12-15 and

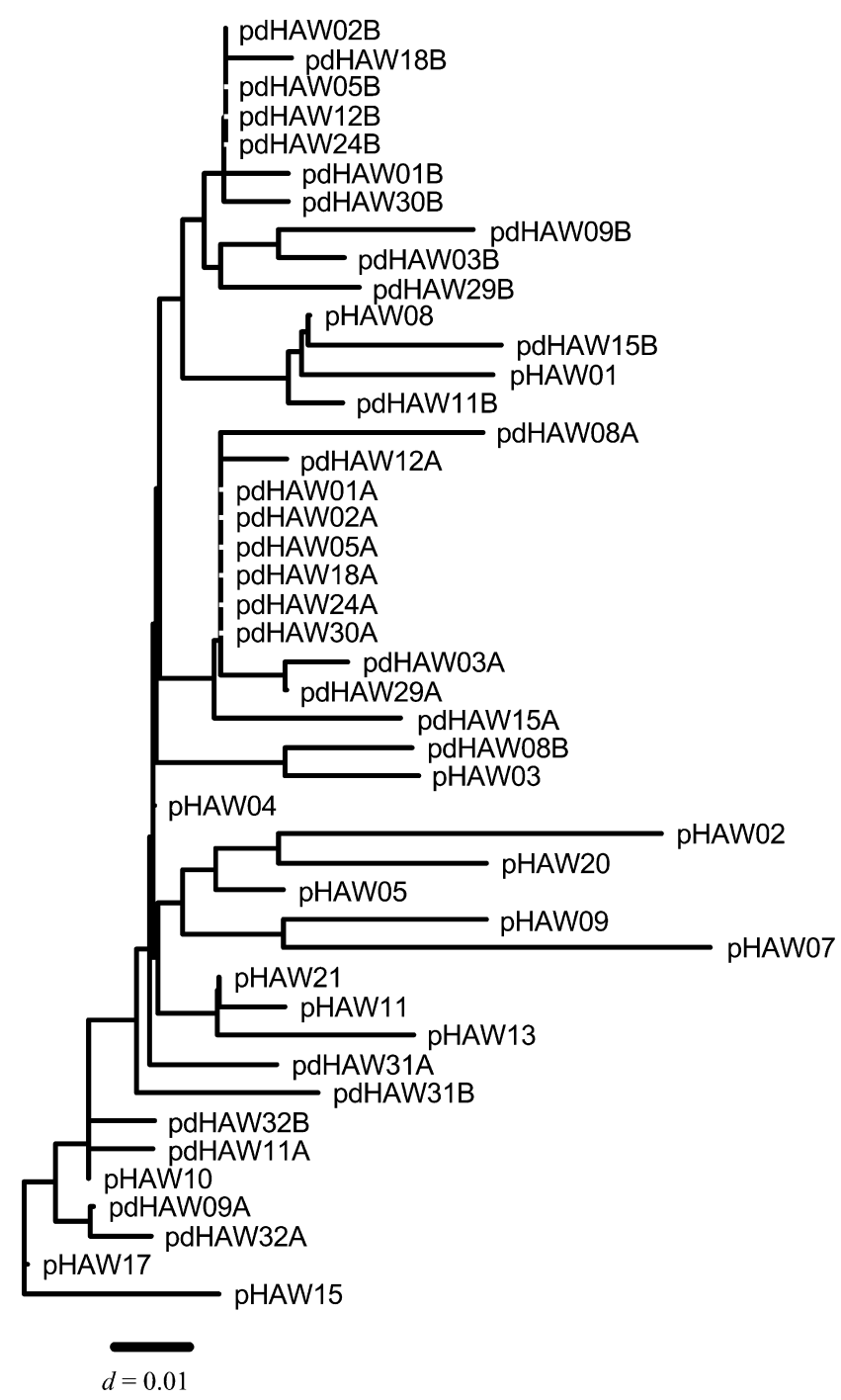

Fig. 3. Unrooted dendogram of the relationship among 45 sequences of T. hawaiensis obtained by the Neighbor-Joining method (accession numbers AJ413515-59). The bar represents genetic distance $d=0.01$ of pair-wise comparisons calculated according to the HKY model. The repeat units cloned as monomers were named pHAW and the repeat units from dimers pdHAW. In each dimer, the repeat unit exhibiting the restriction site Acc I was named pdHAW A, and the unit lacking this site pdHAW B. Bootstrap support values are lower than 50 for all the nodes. 


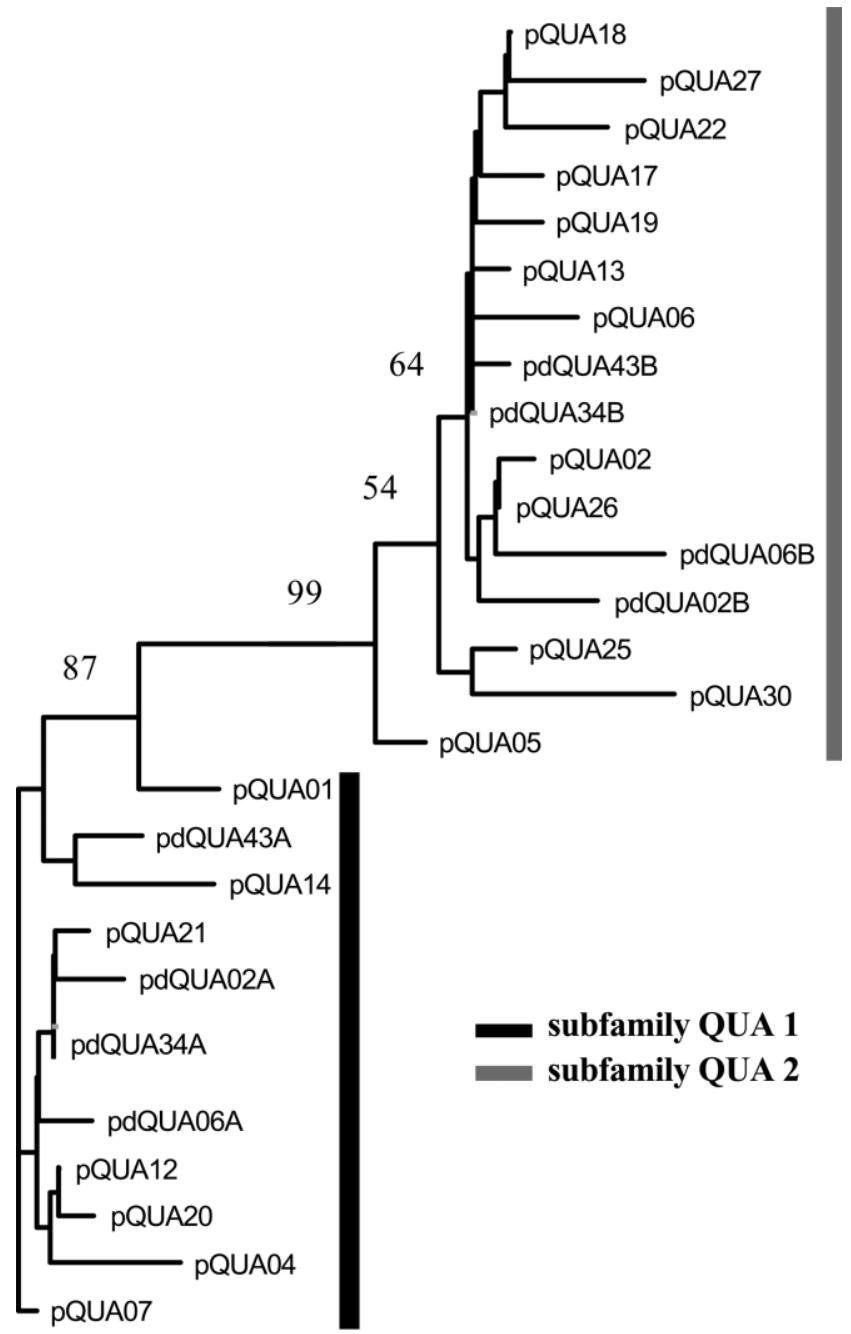

$$
d=0.01
$$

Fig. 4. Unrooted dendogram of the relationships among 27 sequences of T. quasimodo obtained by the Neighbor-Joining method (accession numbers AJ413434-60). The bar represents genetic distance $d=0.01$ of pair-wise comparisons calculated according to the F81 model. Numbers at each node indicate the percentage of trees representing the particular node out of 1000 bootstrap replicates. Bootstrap values under 50 are not indicated. The repeat units cloned as monomers were named pQUA and the repeat units from dimers pdQUA. In each dimer, the repeat unit exhibiting the restriction site $B f a$ I was named pdQUA A, and the unit lacking this site pdQUA B.

175-177). These tracts are the cause of the incomplete homogenization in nine out of the eleven sites between both T. quasimodo subfamilies $(12,15,64,71,76,143,144,175$, and 177). The sequenced dimers of T. quasimodo, composed of one monomer from subfamily QUA 1 (unit pdQUA A) and one from subfamily QUA 2 (unit pdQUA B), have a high degree of sequence identity between dimers (97\%) suggesting that both subfamilies are linked forming a HOR.

The NJ tree inferred from best-fit model $(\mathrm{F} 81+\mathrm{I})$ retrieves three groups of sequences supported by high bootstrap values in T. acuta (Fig. 5): subfamilies ACU 1 (fifteen sequences), ACU 2 (seven sequences), and ACU 3 (eight sequences). Similar groups and bootstrap values were obtained using parsimony. Repetitive units from subfamilies ACU 2 and ACU 3 are 189 bp long but repeats from subfamilies ACU 1 are shorter $(183 \mathrm{bp})$ due to the partial deletion of $6 \mathrm{bp}$ in the $5^{\prime}$ end, out of pACU13 and pACU 18 (both $189 \mathrm{bp}$ ). This deletion very likely occurred during the digestion of the genomic DNA because some repeats have an additional Hinf I recognition site. No other indels are present except for the deletion of a single base in pACU07 (position 125). All T. acuta sequences proved to be homogeneous in their nucleotide composition $(60.33 \% \mathrm{~A}+\mathrm{T}$ rich in average). Moreover, the stDNA sequences of the three Hawaiian Tetragnatha species studied are also homogeneous in their the nucleotide composition.

Nucleotide variability appears to be evenly distributed throughout the monomer but is clearly higher between

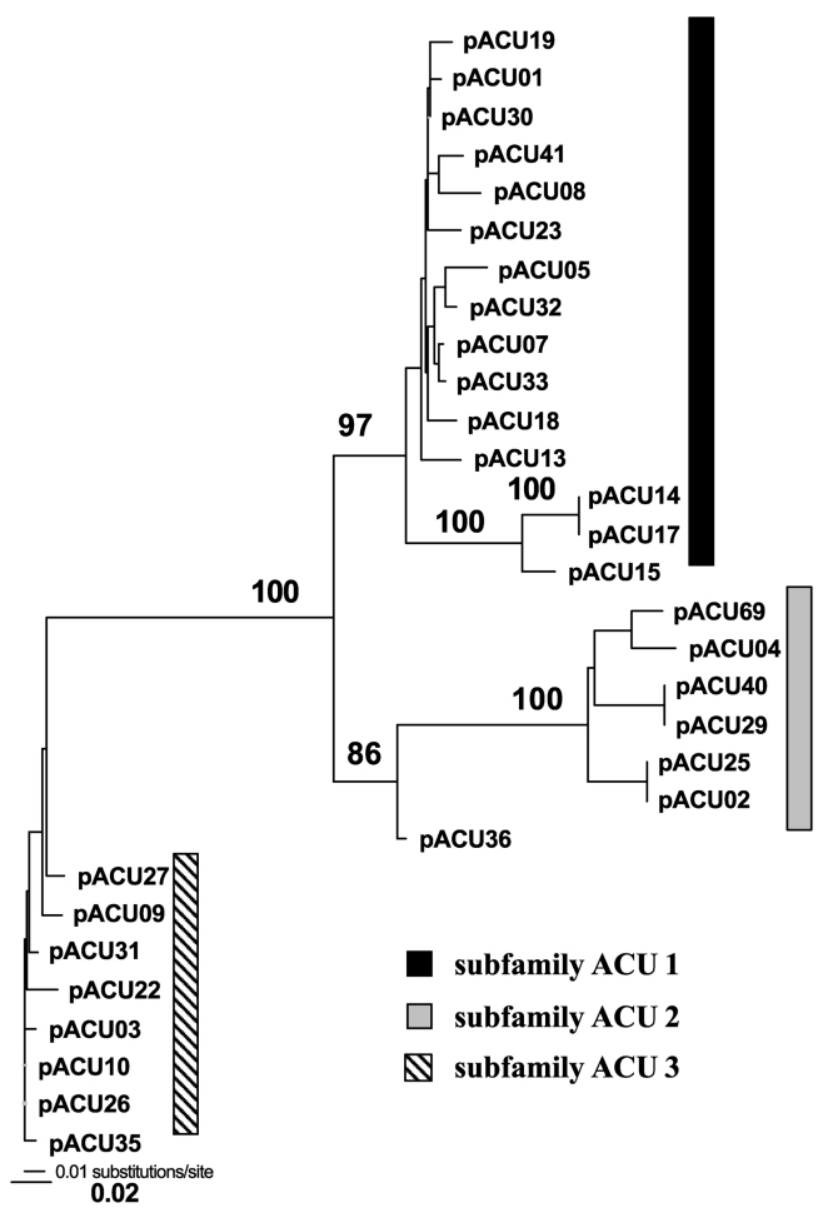

Fig. 5. Unrooted dendogram of the relationships among 30 monomeric sequences of T. acuta obtained by the Neighbor-Joining method (accession numbers AJ413561-76 and AJ511634-46). The bar represents genetic distance $d=0.02$ of pair-wise comparisons calculated according to the F81 + I model. Numbers at each node indicate the percentage of trees representing the particular node out of 1000 bootstrap replicates. Bootstrap values under 50 are not indicated. 
Table 1

Matrix of nucleotide diversity (diagonal), pair-wise divergence values (above the diagonal), and number of fixed sites (below the diagonal) between stDNA subfamilies of $T$. acuta

\begin{tabular}{llcl}
\hline & ACU 1 & ACU 2 & ACU 3 \\
\hline ACU 1 & 0.055 & 0.215 & 0.227 \\
ACU 2 & 11 & 0.080 & 0.274 \\
ACU 3 & 30 & 32 & 0.015 \\
\hline
\end{tabular}

subfamilies than within them (see Table 1). The nucleotide diversity within a particular nucleotide position is mostly due to autapomorphies within the subfamily ACU 3 (ten out of eleven mutations). However, within subfamilies ACU 1 and ACU 2 it is only about 50\% (18 out of 43 and 21 out of 39 , respectively), and the remaining nucleotide diversity is comprised of synapomorphies. Between subfamilies most of the divergence is due to diagnostic nucleotide substitutions (homogenized sites, see Table 1). The number of diagnostic sites between subfamilies ACU 1 and ACU 2 is lower than in the other comparisons. This appears to be due to the sequence pACU36 that exhibits gene conversion tracts (positions 29-36 and 100-114). If this sequence is removed from the analysis, the number of diagnostic sites increases to a similar value to those found in the other comparisons (28 sites). In order to ascertain whether the three subfamilies are forming a HOR, since we could not isolate multimers to clone, we performed digestions of genomic DNA of T. acuta with a battery of restriction enzymes. Some of those endonucleases have a single restriction site which is specific to a single subfamily, and therefore a trimer is expected if one monomer from each one of the three subfamilies are linked forming a HOR: Bfa I (ACU 1), A $f$ II (ACU 2), and Bst BI (ACU 3). The results are inconclusive due the intense smear and/or the weak pattern of the bands (not shown). However, the absence of conspicuous monomers with the three single cutters indicates that repeats of each subfamily are at least not clustered in tandem.

\subsubsection{Interspecific variability}

No cross-hybridization signal was detected among repetitive sequences of $T$. quasimodo, T. hawaiensis or T. acuta (even under low stringency conditions allowing $60 \%$ similarity). However, the alignment of the consensus sequence of the monomers of each species still displays similarity, particularly in some stretches, despite their high divergence (40-45\%, see Fig. 6). To test whether these similarities are statistically significant, we aligned the repetitive consensus sequence of T. hawaiensis with 100 random sequences of the same length and nucleotide composition of the repetitive consensus sequence of T. quasimodo. All pair-wise comparisons between a random sequence of T. quasimodo and the consensus sequence of T. hawaiensis show lower similarities $(30-40 \%)$ than the pair-wise comparison using the original consensus sequences of both species $(55 \%, P<0.01)$. Similar results were obtained when the consensus sequence of $T$. quasimodo was compared with 100 random sequences of T. hawaiiensis, and in all the other pair-wise comparisons (T. quasimodo/T. acuta, and T. hawaiensis/T. acuta). The repetitive sequences of the three Tetragnatha species studied show no significant matches with any sequence in GenBank based on BLAST (Altschul et al., 1997) searches.

The monomers of the three species only have a small number of short perfect direct and inverted sub-repeats up to the size of hexanucleotides. Despite the $\mathrm{A}+\mathrm{T}$ richness of the repeats of the three species they do not show higher frequencies of phased $\mathrm{A}$ or $\mathrm{T}$ runs $\geq 3$ tracts than that expected for random sequences of the same length and nucleotide composition. The repeat units of the three species show neither retarded electrophoretic mobility (not shown) nor specific motifs binding centromeric proteins.

\section{Discussion}

The repeats of three endemic Hawaiian Tetagnatha studied in this paper have similar characteristics to many of the stDNAs described and particularly those in insects (King and Cummings, 1997) with respect to length (140-190 and 300-400 bp) and nucleotide composition (A $+\mathrm{T}$ rich). However, these three spiders studied should be included in the small group of taxa in which this class of DNA constitutes a high fraction of the genome because stDNA usually makes up no more than $10 \%$ of the genome (see reviews by Ugarkovic et al., 1995; Elder and Turner, 1995). Tetragnatha monomers show no potential sub-repeat structures suggesting that these repeats have not originated from smaller sequences as has occurred in insects such Drosophila melanogaster (Lohe et al., 1993).

Despite their marked divergence, the consensus sequences of the monomers of the three Tetragnatha species still display similarity, particularly in some stretches, pointing to a common origin of their repeats. Mutation events and processes of concerted evolution (fixation of speciesspecific stDNA sequences) during speciation have led to the marked interspecific divergences as predicted in the molecular drive model (Dover, 1986). Similar sequences should be found in the other endemic Hawaiian Tetragnatha, and even in the continental lineages that colonized the Hawaiian Islands, since species from the three main Hawaiian clades have stDNA sequences of common origin. The sequences of the repeats are so divergent in the three Tetragnatha taxa that it is not possible to analyze their evolutionary dynamics at the inter-specific level. Therefore, they are not suitable for use as phylogenetic markers at this level, but excellent molecular taxonomic markers at the species level.

The length and nucleotide composition of the repeat units of the three Tetragnatha species seems to be under evolutionary constraint since both are conserved at 


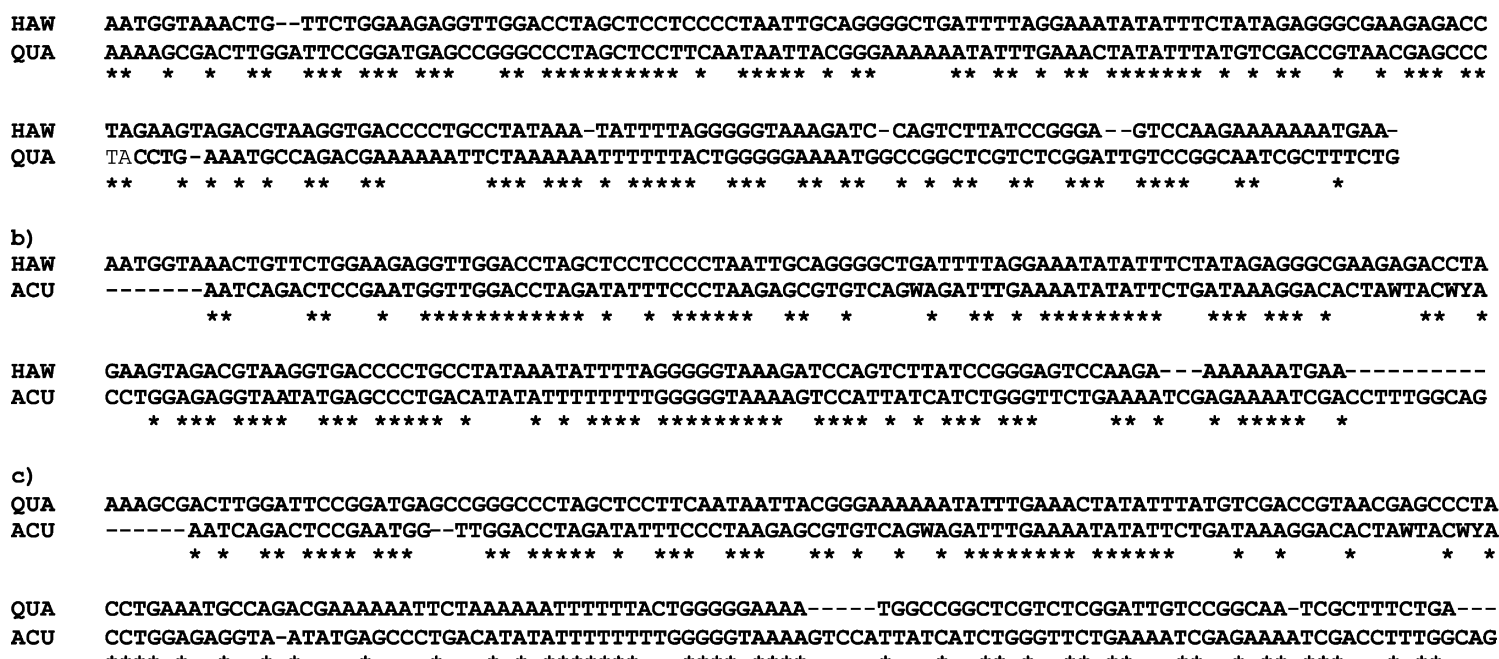

Fig. 6. Pair-wise alignments of the nucleotide sequence of the consensus monomers of T. quasimodo, T. hawaiensis, and T. acuta. Stars denote nucleotide identities between both consensus sequences; dashes denote gaps.

intra- and inter-specific levels. Some authors have suggested that natural selection has an important role in the evolutionary dynamics of repetitive sequences (Stephan and Cho, 1994). This subtle action does not work on the nucleotide sequence per se, but on certain traits of the sequence (e.g. sequence length) that could be critical to their function. In fact, the nucleotide sequence of the Tetragnatha repeat units is very divergent at the inter-specific level (40-45\%) suggesting that, as has been proposed (Stephan and Cho, 1994) and upheld in subsequent studies, the constraints are not acting on the nucleotide sequence. Surprisingly, the length of Tetragnatha repeats and many other stDNAs corresponds closely to the range of nucleosomal length unit suggesting that repeat length could be a critical aspect for the heterochromation condensation and centromeric function and stability (Henikoff et al., 2001). The absence of any of the most common structures associated with stDNA function (phased A or T runs $\geq 3$, cruciform configurations, and specific motifs to bind centromeric proteins) may explain such divergence in nucleotide sequence in the close related Tetragnatha.

At the intra-specific level the three species have different degrees of identity among their repeats. Tetragnatha hawaiensis reveals striking intra-specific uniformity of stDNA sequences suggesting strong homogenization of its monomers. However, it also shows groups of sequences sharing the same nucleotide substitutions within the same site. The occurrence of such shared mutations has been shown in other taxa to be a consequence of the early stages of a gradual spreading of a sequence bearing particular mutations as predicted by the molecular drive model (Strachan et al., 1985; Bachmann and Sperlich, 1993; Pons et al., 2002a). Nevertheless, these mutated sequences are usually lost through strong homogenization because the rate of sexual assortment of chromosomes carrying them in a large population is substantially higher than their rate of spreading by molecular mechanisms of unequal transfer (Dover, 1986).

Contrary to $T$. hawaiensis and most of the described stDNAs, the monomers of T. quasimodo and T. acuta have lower sequence identity mostly due to the coexistence of divergent subfamilies. The presence of subfamilies could be explained by at least three different non-exclusive processes. First, the lack or high bias of unequal transfers between a subset of chromosomes could lead to the fixation of a particular subfamily within each subset of chromosomes (e.g. tse-tse fly, Trick and Dover, 1984). A second possibility is related to the formation of HORs. Theoretical simulations on tandem repetitive sequences indicate that low ratios of unequal crossing over/mutation, common in heterochromatic regions (Charlesworth et al., 1994), tend to form HORs. Thus, these complex and longer repeats are still maintaining the sequence similarity (between higher-orders but not within them) and preserving a repetitive structure. Both characteristics are critical for recombination, which is vital for the survival of the repetitive sequences (Stephan and Cho, 1994). However, the formation and spreading of those HOR units could also be through extra-chromosomal transposition by rolling-circle replication and posterior reinsertion (e.g. stDNA sequences from the genus Ctenomys, Rodentia; Rossi et al., 1990). The latter process is related to population size with the rate of sexual assortment of chromosomes carrying new mutated repeats lower than the rate of spreading of these mutated repeats by unequal transfer leading in small populations to the spreading of several subfamilies (i.e. lower homogenization processes, Dover, 1986).

The results indicate that the two subfamilies of 
T. quasimodo are linked to form a HOR, the dimer. If we consider it as the actual repeat then $T$. quasimodo has a similar striking identity among repeats compared to T. hawaiensis, and therefore also seems to undergo strong homogenization. In the past, T. quasimodo stDNA seems to have reacted to the presence of two divergent monomers creating longer units to keep the sequence similarity, and therefore avoiding their elimination (Stephan and Cho, 1994). On the other hand, the stDNA sequences of T. acuta seems to be under lower homogenization pressure than the other two species, allowing the spreading of different and divergent subfamilies since the organization of the subfamilies of T. acuta in a HOR could not be resolved. The lower sequence identity among $T$. acuta repeats is mostly due to the coexistence of divergent subfamilies but also to lower homogenization within subfamilies, out of subfamily ACU3 (i.e. showing higher numbers of single mutations per sequence or higher numbers of sequences bearing the same mutations). Moreover, evidence of gene conversion or unequal exchange of sister chromatids in T. quasimodo and T. acuta suggests that recombination is more likely to be involved in the spreading and homogenization of stDNA sequences rather than rolling-circle amplification.

In summary, the results suggest evolutionary constraints in sequence length and nucleotide composition of the repeats at the inter-specific level of the Hawaiian Tetragnatha studied but not at the level of the nucleotide sequence. Since the repeats of the three Tetragnatha species probably have a common origin, the different degrees of intra-specific sequence identity, although unclear in some cases, seems to result from traits of the specific organism and/or historical processes that occurred during the speciation process.

\section{Acknowledgements}

This work was funded by a grant from the Obra Cultural i Social Sa Nostra Fundation (Spain) to J.P., with additional funds from the Schlinger Foundation, and National Science Foundation (DEB 9726573) grants to RGG. For help with field work, we are indebted to Haleakala National Park, the Nature Conservancy of Hawaii, Maui Pineapple Company, the State Department of Land and Natural Resources, and the Hawaii Natural Areas Reserve System. For help with collecting we owe particular thanks to M.A. Arnedo, R. Bartlett, C.F. Davenport, J. Giffin, A.C. Medeiros, D. Preston, and G.K. Roderick.

\section{References}

Altschul, S.F., Madden, T.L., Schaeffer, A.A., Zhang, J., Zhang, Z., Miller, W., Lipman, D.J., 1997. Gapped BLAST and PSI-BLAST: a new generation of protein database search programs. Nucleic Acids Res. 25, 3389-3402.
Bachmann, L., Sperlich, D., 1993. Gradual evolution of a specific satellite DNA family in Drosophila ambigua, D. tristis, and D. obscura. Mol. Biol. Evol. 10, 647-659.

Charlesworth, B., Sniegowski, P., Stephan, W., 1994. The evolutionary dynamics of repetitive DNA in eukaryotes. Nature 371, 215-220.

Dover, G., 1986. Molecular drive in multigene families, how biological novelties arise spread and are assimilated. Trends Genet. 2, $159-165$

Elder, J.F., Turner, B.J., 1995. Concerted evolution of repetitive DNA sequences in eukaryotes. Quart. Rev. Biol. 70, 297-323.

Gillespie, R.G., Croom, H.B., Palumbi, S.R., 1994. Multiple origins of a spider radiation in Hawaii. Proc. Natl. Acad. Sci. 91, 2290-2294.

Henikoff, S., Ahmad, K., Malik, H.S., 2001. The centromere paradox, stable inheritance with rapidly evolving DNA. Science 293, 1098-1102.

Higgins, D.G., Thompson, J.D., Gibson, T.J., 1996. Using clustal for multiple sequence alignments. Methods Enzymol. 266, 383-402.

Juan, C., Vazquez, P., Rubio, J.M., Petitpierre, E., Hewitt, G., 1993. Presence of highly repetitive DNA sequences in Tribolium flourbeetles. Heredity 70, 1-8.

King, L.M., Cummings, M.P., 1997. Satellite DNA repeat sequence variation is low in three species of burying beetles in the genus Nicrophorus Coleoptera. Silphidae. Mol. Biol. Evol. 14, $1088-1095$.

Lohe, A.R., Hilliker, A.J., Roberts, P.A., 1993. Mapping simple repeated DNA sequences in heterochromatin of Drosophila melanogaster. Genetics 134, 1149-1174.

Lorite, P., Carrillo, J.A., Tinaut, A., Palomeque, T., 2002. Comparative study of satellite DNA in ants of the Messor genus. Gene 297, $113-122$.

Mantovani, B., Tinti, F., Bachmann, L., Scalli, V., 1997. The Bag 320 satellite DNA family in Bacillus stick insects Phasmatodea: different rates of molecular evolution of highly repetitive DNA in bisexual and parthenogenetic taxa. Mol. Biol. Evol. 14, 1197-1205.

Martinez-Balbas, A., Rodriguez-Campos, A., Garcia-Ramirez, M., Sainz, J., Carrera, P., Aymani, J., Azorin, F., 1990. Satellite DNAs contain sequences that induce curvature. Biochemistry 29, 2342-2348.

Miklos, G.L., Gill, A.C., 1981. The DNA sequences of cloned complex satellite DNAs from Hawaiian Drosophila and their bearing on satellite DNA sequence conservation. Chromosoma 82, 409-427.

Modi, W.S., Gallagher, D.S., Womack, J.E., 1996. Evolutionary histories of highly repeated DNA families among the Artiodactyla Mammalia. J. Mol. Evol. 42, 337-349.

Pons, J., Petitpierre, E., Juan, C., 2002a. Evolutionary dynamics of satellite DNA family PIM357 in species of the genus Pimelia (Tenebrionidae Coleoptera). Mol. Biol. Evol. 19, 1329-1340.

Pons, J., Petitpierre, E., Juan, C., 2002b. Higher-order organisation and compartmentalization of satellite DNA PIM357 in species of the coleopteran genus Pimelia. Chromosome Res. 10, 597-606.

Posada, D., Crandall, K.A., 1998. MODELTEST: testing the model of DNA substitution. Bioinformatics 14, 817-818.

Rice, P., Longden, I., Bleasby, A., 2000. EMBOSS: The European Molecular Biology Open Software Suite. Trends Genet. 16, 276-277.

Rossi, M.S., Reig, O.A., Zorzopulos, J., 1990. Evidence for rolling-circle replication in a major satellite DNA from the South American rodents of the genus Ctenomys. Mol. Biol. Evol. 7, 340-350.

Rozas, J., Rozas, R., 1997. DnaSP version 2.0 a novel software package for extensive molecular population genetic analysis. Comput. Applic. Biosci. 13, 307-311.

Stephan, W., Cho, S., 1994. Possible role of natural selection in formation of tandem-repetitive noncoding DNA. Genetics 136, 333-341.

Strachan, T., Webb, D., Dover, G., 1985. Transition stages of molecular drive in multiple-copy DNA families in Drosophila. EMBO J. 4, $1701-1708$.

Swofford, D.L, 2002. PAUP*. Phylogenetics Analysis Using Parsimony *and other methods. Version 4.05, edited by Sinauer Associates, Sunderland, MA 
Trick, M., Dover, G., 1984. Unexpectedly slow homogenization within a repetitive DNA family shared between two subspecies of tsetse fly. J. Mol. Evol. 20, 322-329.

Ugarkovic, D., Plohl, M., 2002. Variation in satellite DNA profiles-causes and effects. EMBO J. 21, 5955-5959.
Ugarkovic, D., Petitpierre, E., Juan, C., Plohl, M., 1995. Satellite DNAs in tenebrionid species: structure, organization and evolution. Croat. Chem. Acta 68, 627-638.

Willard, H.F., Wayne, J.S., 1987. Hierarchical order in chromosomespecific human alpha satellite DNA. Trends Genet. 3, 192-198. 